\title{
A COMPARATIVE STUDY OF DIMENSION REDUCTION TECHNIQUES FOR CONTENT-BASED IMAGE RETRIEVAL
}

\author{
G. Sasikala ${ }^{1}$, R. Kowsalya ${ }^{2}$, Dr. M. Punithavalli ${ }^{3}$ \\ ${ }^{1,2}$ GR Govindarajulu School of Applied Computer Technology, \\ PSGR Krishnammal College for Women, Coimbatore, Tamil Nadu \\ $\frac{\text { sasikala@grgsact.com }}{\text { Kowsalyadgrgsact.com }}$
}

${ }^{3}$ Sri Ramakrishna College of Arts \& Science for Women, New Siddhapudur, Coimbatore - 641044.

\begin{abstract}
Efficient and effective retrieval techniques of images are desired because of the explosive growth of digital images. Content-based image retrieval is a promising approach because of its automatic indexing and retrieval based on their semantic features and visual appearance. This paper discusses the method for dimensionality reduction called Maximum Margin Projection (MMP). MMP aims at maximizing the margin between positive and negative sample at each neighborhood. It is designed for discovering the local manifold structure. Therefore, MMP is likely to be more suitable for image retrieval systems, where nearest neighbor search is usually involved. The performance of these approaches is measured by a user evaluation. It is found that the MMP based technique provides more functionalities and capabilities to support the features of information seeking behavior and produces better performance in searching images.
\end{abstract}

\section{KEYWORDS}

Content Based Image Retrieval, Maximum Margin Subspace, Dimensionality Reduction.

\section{INTRODUCTION}

Content based image retrieval is motivated by the fast growth of digital image databases [1],[2]. This in turn requires efficient search schemes. The low-level visual features (color, texture, shape, etc.,) are routinely extracted to symbolize the images. However, the low-level features may not precisely characterize the high-level semantic concepts. To narrow down this semantic gap, relevance feedback is introduced into CBIR [3].

Various techniques have been developed for reducing the dimensionality of the feature space, in the anticipation of obtaining a more controllable problem. The most popular dimensionality reduction algorithms include Principal Component Analysis (PCA) [4],[5] and Linear Discriminant Analysis (LDA) [4],[6]. Both PCA and LDA have extensively been applied to image retrieval, face recognition, information retrieval and pattern recognition. However, they are intended for discovering only the global Euclidean structure, whereas the local manifold 
structure is unnoticed. The global statistics such as variance is often complicated to approximate when there are no adequate samples.

In this paper a method called Maximum Margin Projection (MMP), which focuses on local discriminant analysis for image retrieval is compared with Local Discriminant Analysis (LDA). In MMP both geometrical and discriminant structures of the data manifold are considered.

\section{Motivation}

Image retrieval is a challenging issue in the real world applications. Many algorithms have been proposed by researchers for image retrieval. This section of the paper presents an overview of algorithms proposed by researchers for effective image retrieval.

The curse of dimensionality has a direct bearing on similarity retrieval (i.e., finding nearest neighbors) in high dimensions in the sense that if sense that it raises the issue of whether or not nearest neighbor searching is even meaningful in such an environment. In particular, it has been shown that for data and queries drawn from a uniform distribution, the distance to the nearest neighbor and the distance to the farthest neighbor tend to coverage as the dimension increases [18].

A number of methods have been proposed to overcome the curse of dimensionality. One approach is to observe that the data is rarely uniformly distributed which leads to pointing out that some dimensions are more significant than others thereby focussing on them (e.g., [18, 19, and 20]). Such methods are also known as dimension-reduction techniques and some examples include SVD [21] and Discrete Fourier Transform (DFT) [22]. The traditional and the state-ofthe-art dimensionality reduction methods can be generally classified into the feature extraction $[23,24,25]$ and feature selection [26, 27, 28] approaches. In general, feature extraction approaches are more effective than feature selection techniques [29, 30, 31] and they have shown to be very effective for real-world dimensionality reduction problems [23, 24, 32, 33]. Many scalable online FE algorithms have been proposed.

Incremental PCA (IPCA) [34, 35] is a well-suited incremental learning algorithm. The latest version of IPCA is called Candid Covariance-free Incremental Principal Component Analysis (CCIPCA) [36]. However, IPCA ignores the valuable label information of data and is not optimal for general classification tasks. The incremental Linear Discriminant Analysis (ILDA) [37] algorithm has been proposed. Another feature extraction algorithm is called Incremental Maximum Margin Criterion (IMMC) [38].

Wang et al. proposed a nonlinear manifold embedding method [7] for Image retrieval. A novel semi-supervised learning method for dimensionality reduction, namely kernel maximum margin projection (KMMP) is proposed in this paper. This approach addresses an issue in practical applications in which the limited number of user feedback is usually overwhelmed by the large number of dimensionalities of the visual feature space. KMMP is designed for discovering the local manifold structure. After projecting the images into a lower dimensional subspace, KMMP significantly improves the performance of image retrieval.

A posterior pseudo-probability function based on Bayes' formula [8] has been described by Xiabi Liu et al. This approach transforms class-conditional probability values into posterior class probability values. The parameters in this function are trained from the data using the proposed max-min posterior pseudo-probability method in which the optimization objective is to 
maximize the posterior pseudo-probabilities for positive samples, at the same time to minimize those for negative samples. By using the MMP training method, the posterior pseudo-probability function of each class is produced to reflect the maximum separability between it and other classes.

William Robson Schwartz and Hélio Pedrini together put forth an approach for color textured image segmentation [9]. This paper presents a new image segmentation method using color texture features extracted from 3D co-occurrence matrices combined with spatial dependence, this modeled by a Markov random field. The 3D co-occurrence matrices provide features which summarize statistical interaction both between pixels and different color bands, which is not usually accomplished by other segmentation methods. After a preliminary segmentation of the image into homogeneous regions, the ICM method is applied only to pixels located in the boundaries between regions, providing a fine segmentation with a reduced computational cost, since a small portion of the image is considered in the last stage.

\section{MAXIMUM MARGIN PROJECTION FOR CBIR}

This section explains the MMP algorithm which considers both discriminant and geometrical structures in the data and also the implementation of the MMP algorithm for image retrieval. The algorithm shares some common properties with some recent work on combining classification and metric learning such as Distance-Function Alignment $\left(\mathrm{D}_{\text {Align }}\right)$ [14] and Spectral Kernel Learning (SKL) [15].

Low-level image depiction is a fundamental problem in CBIR. General visual features comprise color, texture, shape, etc. Color and texture features are the most comprehensively used visual features in CBIR. Therefore dimensionality reduction is important problem in CBIR. The generic problem of linear dimensionality reduction is explained as follows: Given a set $\mathrm{x}_{1}, \ldots, \mathrm{x}_{\mathrm{m}}$ in $\operatorname{IR}^{\mathrm{n}}$, find a transformation matrix $\mathrm{A}=\left(\mathrm{a}_{1}, \ldots, \mathrm{a}_{\mathrm{d}}\right)$ that maps these $\mathrm{m}$ points to a set of points $y_{1}, \ldots, y_{m}$ in $I^{d}(d<<n)$ such that $y_{i}$ "represents" $x_{i}$, where $y_{i}=A^{T} x_{i}$.

This paper considers the issue of maximizing a local margin between relevant and irrelevant images. In image retrieval, the labeled images include the original query image and the images with user's relevance feedback. The problem of image retrieval concerns ranking the unlabeled images according to their relevance to the original query image. Relevance feedback is one of the most important techniques for narrowing down the gap between low-level visual features and high-level semantic concepts [3]. The typical retrieval process is outlined as follows the user submits a query image example to the system. The system ranks the images in the database according to some predefined distance metric and presents to the user the top ranked images. The user provides the relevance feedback to the system by labeling images as "relevant" or "irrelevant." The system uses the user-provided information to rerank the images in the database and returns the top images to the user.

The user's relevance feedback can be used to update the within-class and between-class graphs for discovering the semantic and geometrical structure of the image database for effective image retrieval. In order to model the local geometrical structure it is necessary to construct a nearest neighbor graph $\mathrm{G}$. The nearest neighbor graph $\mathrm{G}$ with weight matrix $\mathrm{W}$ characterizes the local geometry of the data manifold. It has frequently been used in manifold-based learning techniques such as [10], [11], [12], [13].

At the initial stage of the retrieval the user submits a query image q. The images in the database are ranked according to the Euclidean distances $\&$ the top images are presented to the 
user. The user is then required to mark the top returned images as "relevant" or "irrelevant". Naturally, the images can be divided into two classes based on this relevance feedback. We can construct the within-class and between-class graphs. At the beginning of the retrieval there is no relevance feedback available. Thus, by our definition the within-class graph is simply G, whereas the between-class graph is an empty graph. During every iteration of relevance feedback the only need is to update the within-class and between-class graphs according to

$$
\begin{aligned}
& \mathrm{W}_{\mathrm{b}, \mathrm{ij}}=\left\{\begin{array}{l}
1, \text { if } \mathrm{X}_{\mathrm{i}} \varepsilon \mathrm{N}_{\mathrm{b}}\left(\mathrm{X}_{\mathrm{j}}\right) \text { or } \mathrm{X}_{\mathrm{j}} \varepsilon \mathrm{N}_{\mathrm{b}}\left(\mathrm{X}_{\mathrm{i}}\right), \\
0, \text { otherwise. }
\end{array}\right. \\
& \mathrm{W}_{\mathrm{w}, \mathrm{ij}}= \begin{cases}\gamma, & \text { if } \mathrm{X}_{\mathrm{i}} \text { and } \mathrm{X}_{\mathrm{j}} \text { share the same label, } \\
1, & \text { if } \mathrm{X}_{\mathrm{i}} \text { or } \mathrm{X}_{\mathrm{j}} \text { is unlabeled } \\
\text { but } \mathrm{X}_{\mathrm{i}} \varepsilon \mathrm{N}_{\mathrm{w}}\left(\mathrm{X}_{\mathrm{j}}\right) \text { or } \mathrm{X}_{\mathrm{j}} \varepsilon \mathrm{N}_{\mathrm{w}}\left(\mathrm{X}_{\mathrm{i}}\right), \\
0, & \text { otherwise. }\end{cases}
\end{aligned}
$$

By applying this MMP algorithm the images can be projected into a lower dimensional subspace in which semantically related images tend to be close to each other.

When MMP is applied one needs to estimate the optimal dimensionality of the subspace. It would be important to note that the MMP algorithm is inherently a graph embedding algorithm. It is reliably associated to Laplacian Eigenmaps [10], LLP [11], spectral clustering [16] and Normalized Cut [17].

\section{EXPERIMENTAL RESULTS}

In this section the performance of MMP algorithm is evaluated on COREL dataset. It is a large and heterogeneous image set. Each image is represented as a 128-dimensional vector. To demonstrate the effectiveness of this image retrieval algorithm (MMP) it is compared with the state of art algorithm LDA. After the user provides relevance feedback, the LDA and MMP algorithms are then applied to rerank the images in the database. Figure.1 represents the comparison of the MMP algorithm and LDA algorithm. The experimental results reveal that MMP algorithm performs better than LDA. The comparison chart represents that the precision value of the MMP algorithm increases for each iteration.

The actual computational time of the MMP algorithm and LDA algorithm for processing one query is given in Table 1 . These two algorithms can respond to the user's query very fast approximately $0.1 \mathrm{~s}$. 
TABLE 1 : Average runtime of MMP \& LDA algorithms for processing one query

\begin{tabular}{|c|c|c|c|}
\hline \multirow{2}{*}{ Algorithm } & \multicolumn{3}{l|}{ Time at different feedback iterations (s) } \\
\cline { 2 - 4 } & 1 & 2 & 3 \\
\hline MMP & 0.066 & 0.071 & 0.075 \\
\hline LDA & & & \\
\hline
\end{tabular}

(a) Feedback iteration 1

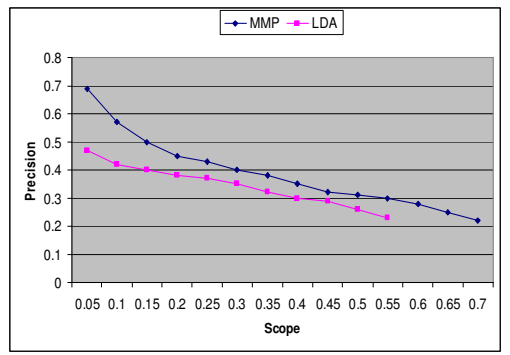

(b) Feedback iteration 2

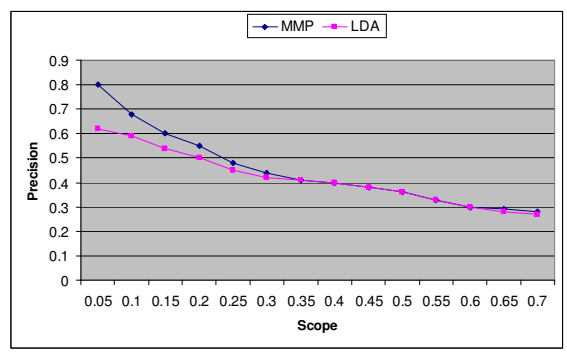

Figure.1 The average precision-scope curves of different algorithms for the first two feedback iterations. The MMP algorithm performs the best on the entire scope.

\section{CONCLUSION}

This paper presents a manifold learning algorithm called MMP for image retrieval. In the first step a between-class nearest neighbor graph and a within-class nearest neighbor graph to model both geometrical and discriminant structures in the data are constructed. This paper considered the image retrieval problem on a small, static and closed-domain image data. The experimental results validate that the MMP achieves a significantly higher precision. In future enhancements it would be very interesting to explore different ways of constructing the image graph to model the semantic structure in the data

\section{REFERENCES}

[1] J. Bi, Y. Chen and J.Z. Wang, "A Sparse Support Vector Machine Approach to Region-Based Image Categorization," Proc. IEEE Conf. Computer Vision Pattern Recognition (CVPR '04), 2004.

[2] E. Chang, K. Goh, G. Sychay \& G. Wu, "Cbsa: Content-Based Soft Annotation for Multimodal Image Retrieval Using Bayes Point Machines," IEEE Trans. Circuits \& Systems for Video Tech., vol. 13, no. 1, pp. 26-38, Jan. 2003. 
[3] Y. Rui, T.S. Huang, M. Ortega and S. Mehrotra, "Relevance Feedback: A Power Tool for Interactive Content-Based Image Retrieval," IEEE Trans. Circuits and Systems for Video Technology, vol. 8, no. 5, 1998.

[4] R.O. Duda, P.E. Hart and D.G. Stork, Pattern Classification, second ed. Wiley-Interscience, 2000.

[5] Z. Su, S. Li and H.-J. Zhang, "Extraction of Feature Subspace for Content-Based Retrieval Using Relevance Feedback," Proc. Ninth Ann. ACM Int'l Conf. Multimedia (Multimedia '01), 2001.

[6] D.L. Swets and J. Weng, "Using Discriminant Eigenfeatures for Image Retrieval," IEEE Trans. Pattern Analysis and Machine Intelligence, vol. 18, no. 8, pp. 831-836, Aug. 1996.

[7] Can Wang, Jun Zhao, Xiaofei He, Chun Chen and Jiajun Bu, "Image retrieval using nonlinear manifold embedding," Neurocomputing, Volume 72, Issues 16-18, October 2009, Pages 3922 - 3929, Financial Engineering; Computational \& Ambient Intelligence (IWANN 2007).

[8] Xiabi Liu, Yunde Jia, Xuefeng Chen, Yuan Deng and Hui Fu, "Max-Min Posterior PseudoProbabilities Estimation of Posterior Class Probabilities to Maximize Class Separability," IEEE Transactions on Speech and Audio Processing, vol. 5, no. 3, pp. 257-265, 1997.

[9] William Robson Schwartz and Hélio Pedrini, "Color Textured Image Segmentation Based on Spatial Dependence Using 3D Co-occurrence Matrices \& Markov Random Fields," IEEE Transaction on Medical Imaging, vol.15, no.6, pp.871-880, December 1996.

[10] M. Belkin and P. Niyogi, "Laplacian Eigenmaps and Spectral Techniques for Embedding \& Clustering," Advances in Neural Information Processing Systems 14, pp. 585-591, MIT Press, 2001.

[11] X. He and P. Niyogi, "Locality Preserving Projections," Advances in Neural Information Processing Systems 16, MIT Press, 2003.

[12] S. Roweis and L. Saul, "Nonlinear Dimensionality Reduction by Locally Linear Embedding," Science, vol. 290, no. 500, pp. 2323- 2326, 2000.

[13] J. Tenenbaum, V. de Silva and J. Langford, "A Global Geometric Framework for Nonlinear Dimensionality Reduction,” Science, vol. 290, no. 500, pp. 2319-2323, 2000.

[14] G. Wu, E.Y. Chang and N. Panda, "Formulating Context-Dependent Similarity Functions," Proc. 13th Ann. ACM Int'l Conf. Multimedia (Multimedia '05), Nov. 2005.

[15] S.C.H. Hoi, M.R. Lyu and E.Y. Chang, "Learning the Unified Kernel Machines for Classification," Proc. 12th ACM SIGKDD Int'l Conf. Knowledge Discovery and Data Mining, pp. 187-196, 2006.

[16] A.Y. Ng, M. Jordan and Y. Weiss, "On Spectral Clustering: Analysis and an Algorithm," Advances in Neural Information Processing Systems 14, pp. 849-856, MIT Press, 2001.

[17] J. Shi and J. Malik, "Normalized Cuts and Image Segmentation", IEEE Trans. Pattern Analysis and Machine Intelligence, vol. 22, no. 8, pp. 888-905, Aug. 2000.

[18] P. Indyk and R. Motwani. Approximate nearest neighbors: Towards removing the curse of dimensionality. In Proceedings of the $30^{\text {th }}$ Annual ACM Symposium on the Theory of Computing, Pages 604-613, Dallas, May 1998.

[19] A. Gionis, P. Indyk and R. Motwani. "Similarity search in high dimensions via hashing". In Proceedings of the $25^{\text {th }}$ International Conference on Very Large Data Bases ( VLDB), M. P. 
Atkinson, M. E. Orlowska, P. Valduriez, S. B. Zdonik, and M. L. Brodie, eds., pages 518529, Edinburgh, Scotland, September 1999.

[20] A. Hinneburg, C. C. Aggarwal, and D. A. Kevin. What is the nearest neighbour in high dimensional spaces. In Proceedings of the $26^{\text {th }}$ International Conference on very large Data Bases (VLDB ), A. El Abbadi, M. L. Brodie, S. Chakravarthy, U. Dayal, N. Kamel, G. Schlageter, and K. Y . Whang, eds., pages 506-515, Cairo, Egypt, September 2000.

[21] G. H. Golub and C. F. van Loan. Matrix Computations. Johns Hopkins University Press, Baltimore, MD, third edition, 1996.

[22] N. Gershenfeld. The Nature of Mathematical Modelling. Cambridge University Press, Cambridge, United Kingdom, 1999.

[23] D.D. Lewis, "Feature Selection and Feature Extraction for Text Categorization," Proc. Workshop Speech and Natural Language, pp.212-217, 1992.

[24] H. Li, T. Jiang, and K. Zhang, "Efficient and Robust Feature Extraction by Maximum Margin Criterion," Proc. Conf. Advances in Neural Information Processing Systems, pp. 97-104, 2004.

[25] E. Oja, "Subspace Methods of Pattern Recognition," Pattern Recognition and Image Processing Series, vol. 6, 1983.

[26] A.L. Blum and P. Langley, "Selection of Relevant Features and Examples in Machine Learning," Artificial Intelligence, vol. 97, nos.1-2, pp. 245-271, 1997.

[27] K. Daphne and M. Sahami, "Toward Optimal Feature Selection," Proc. 13th Int'l Conf. Machine Learning, pp. 284-292, 1996.

[28] Y. Yang and J.O. Pedersen, "A Comparative Study on Feature Selection in Text Categorization,” Proc. 14th Int'l Conf. Machine Learning, pp. 412-420, 1997.

[29] R.O. Duda, P.E. Hart, and D.G Stork, Pattern Classification, second ed. John Wiley, 2001.

[30] A.R. Webb, Statistical Pattern Recognition, second Ed. John Wiley, 2002.

[31] J. Yan, N. Liu, B.Y. Zhang, S.C. Yan, Q.S. Cheng, W.G. Fan, Z. Chen, W.S. Xi, and W.Y. Ma, "OCFS: Orthogonal Centroid Feature Selection," Proc. 28th Ann. Int'l ACM SIGIR Conf. Research and Development in Information Retrieval, 2005.

[32] W. Fan, M.D. Gordon, and P. Pathak, "Effective Profiling Of Consumer Information Retrieval Needs: A Unified Framework and Empirical Comparison," Decision Support Systems, vol. 40, pp. 213-233, 2004.

[33] R. Hoch, "Using IR Techniques for Text Classification in Document Analysis," Proc. 17th Ann. Int'l ACM SIGIR Conf. Research and Development in Information Retrieval, pp. 3140, 1994.

[34] M. Artae, M. Jogan, and A. Leonardis, "Incremental PCA for On- Line Visual Learning and Recognition,” Proc. 16th Int'l Conf. Pattern Recognition, pp. 781-784, 2002.

[35] Y. Li, L. Xu, J. Morphett, and R. Jacobs, "An Integrated Algorithm of Incremental and Robust PCA,” Proc. Int'l Conf. Image Processing, pp. 245-248, 2003.

[36] J. Weng, Y. Zhang, and W. S. Hwang, "Candid Covariance-Free Incremental Principal Component Analysis," IEEE Trans. Pattern Analysis and Machine Intelligence, vol. 25, pp. 1034-1040, 2003. 
[37] K. Hiraoka, K. Hidai, M. Hamahira, H. Mizoguchi, T. Mishima, and S. Yoshizawa, "Successive Learning of Linear Discriminant Analysis: Sanger-Type Algorithm," Proc. 14th Int'1 Conf. Pattern Recognition, pp. 2664-2667, 2000.

[38] J. Yan, B.Y. Zhang, S.C. Yan, Z. Chen, W.G. Fan, Q. Yang, W.Y. Ma and Q.S. Cheng, "IMMC: Incremental Maximum, Marginal Criterion," Proc. 10th ACM SIGKDD Int'l Conf. Knowledge Discovery and Data Mining, pp. 725-730, 2004.

[39] Xiaofei He, Deng Cai and Jiawei Han, "Learning a Maximum Margin Subspace for Image Retrieval", IEEE Trans. On Knowledge and Data Engineering", Vol. 20, No. 2, February 2008.

[40] Dave Tahmoush and Hanan Samet, "High-Dimensional Similarity Retrieval Using Dimensional Choice", University of Maryland, First International Workshop on Similarity Search and Applications.

\section{Authors}

1.G. Sasikala, Lecturer, GR Govindarajulu School of Applied Computer Technology, PSGR Krishnammal College for Women, Coimbatore, Tamil Nadu

2.R. Kowsalya, Lecturer, GR Govindarajulu School of Applied Computer Technology, PSGR Krishnammal College for Women,

Coimbatore, Tamil Nadu

3.Dr. M. Punithavalli, Director, Department of Computer Science, Sri Ramakrishna College of Arts \& Science for Women, 395, Sarojini Naidu Road, New Siddhapudur, Coimbatore - 641044. 